\title{
A NEW PROOF OF ALMOST ISOMETRY THEOREM IN ALEXANDROV GEOMETRY WITH CURVATURE BOUNDED BELOW*
}

\author{
YUSHENG WANG ${ }^{\dagger}$, XIAOLE SU ${ }^{\ddagger}$, AND HONGWEI SUN ${ }^{\S}$
}

\begin{abstract}
In this paper we give a new proof (along the line of the original proof) for the almost isometry theorem in Alexandrov geometry with curvature bounded below in [1]. The motivation of the new proof is that we find that Lemma 9.11 in [1] is incorrect (see Example 1.3 below), while this lemma is a crucial step in the original proof.
\end{abstract}

Key words. Alexandrov spaces, GH-approximation, almost isometry.

AMS subject classifications. 53-C20.

In the seminal paper [1] on Alexandrov geometry with curvature bounded below, the almost isometry theorem (see Theorem 0.1 below) asserts (roughly) that if the Gromov-Hausdorff distance between two compact $n$-dimensional Alexandrov spaces with the same low curvature bound is sufficiently small and if one of them contains only $(n, \delta)$-strained points, then there is an almost isometry $\bar{h}$ between the two spaces. We think that, in the original proof of the almost isometry theorem, Lemma 9.11 in [1] (Lemma 1.2 below) is incorrect (see Example 1.3 below). Precisely, the condition (1.1) of Lemma 9.11 is inadequate for its conclusion. An important part of the original proof of the almost isometry theorem is to verify that $\bar{h}$ satisfies (1.1). In our new proof we replace the condition (1.1) with (2.3), and show that the conclusion of Lemma 9.11 still holds (see Lemma 2.1 below). Moreover, additional work is required to verify that $\bar{h}$ satisfies (2.3) (via locally constructing an almost isometry using Lemma 2.1, and proving that the local map is sufficiently close to $\bar{h}$ ).

0. Notations and main theorem. We first collect some notations almost all of which come from [1].

- $|x y|$ always denotes the distance between two points $x$ and $y$ in a metric space.

- For any three points $p, q, r$ in a length space, we associate a triangle $\triangle \tilde{p} \tilde{q} \tilde{r}$ on the $k$-plane (2-dimensional complete and simply-connected Riemannian manifold of constant curvature $k$ ) with $|\tilde{p} \tilde{q}|=|p q|,|\tilde{p} \tilde{r}|=|p r|$ and $|\tilde{r} \tilde{q}|=|r q|$. For $k \leqslant 0$ and for $k>0$ with $|p q|+|p r|+|q r| \leqslant 2 \pi / \sqrt{k}$, such a triangle always exists. We denote by $\tilde{\angle} p q r$ the angle of the triangle $\triangle \tilde{p} \tilde{q} \tilde{r}$ at vertex $\tilde{q}$.

- $M$ always denotes an Alexandrov space with curvature bounded below by $k$, which is a length space and in which there exists a neighborhood $U_{x}$ of any $x \in M$ such that for any four (distinct) points $(a ; b, c, d)$ in $U_{x}$

$$
\tilde{\angle} b a c+\tilde{\angle} b a d+\tilde{\angle} c a d \leqslant 2 \pi .
$$

- A point $p \in M$ is called an $(n, \delta)$-strained point if there are $n$ pairs of points

\footnotetext{
*Received February 5, 2012; accepted for publication July 26, 2012.

${ }^{\dagger}$ Corresponding author. School of Mathematical Sciences (and Lab. Math. Com. Sys.), Beijing Normal University, Beijing, 100875 P.R.C. (wwyusheng@gmail.com). Supported by NSFC 11001015 and 11171025 .

${ }^{\ddagger}$ School of Mathematical Sciences (and Lab. Math. Com. Sys.), Beijing Normal University, Beijing, 100875 P.R.C. (suxiaole@bnu.edu.cn). Supported by NSFC 10801011 and 11171025.

$\S$ School of Mathematical Sciences, Capital Normal University, Beijing, 100037 P.R.C. (hwsun@ bnu. edu.cn).
} 
distinct from $p,\left(a_{i}, b_{i}\right)$, such that for $i \neq j$

$$
\begin{array}{r}
\tilde{L} a_{i} p b_{i}>\pi-\delta, \tilde{\angle} a_{i} p a_{j}>\pi / 2-\delta, \\
\tilde{L} a_{i} p b_{j}>\pi / 2-\delta, \tilde{L} b_{i} p b_{j}>\pi / 2-\delta .
\end{array}
$$

$\left\{\left(a_{i}, b_{i}\right)\right\}_{i=1}^{n}$ is called an $(n, \delta)$-strainer at $p$ (which is obviously a generalization of a coordinate frame). We say that the $(n, \delta)$-strainer $\left\{\left(a_{i}, b_{i}\right)\right\}_{i=1}^{n}$ at $p$ is $R$-long if $\left|a_{i} p\right|>\frac{R}{\delta}$ and $\left|b_{i} p\right|>\frac{R}{\delta}$ for all $i$. And we denote by $M(n, \delta, R)$ the set of points with $R$-long $(n, \delta)$-strainer in $M$.

- An important fact is that if any neighborhood of a point $p \in M$ contains an $(n, \delta)$-strained point ( $\delta$ is sufficiently small) but no $(n+1, \delta)$-strained point, then any neighborhood of any other point in $M$ has the same property (see $\S 6$ in [1]). And it follows that the dimension of such $M$ is defined to be $n$.

- We always denote by $\varkappa(\cdot)$ or $\varkappa(\cdot, \cdot)$ (resp. $C$ ) a positive function which is infinitesimal at zero (e.g. $\varkappa\left(\delta, \delta_{1}\right) \longrightarrow 0$ as $\left.\delta, \delta_{1} \longrightarrow 0\right)$ (resp. a constant depending only on $n$ ); however we do not distinguish any two $\varkappa$-functions with the same parameters (resp. any two such constants) when we use them.

- A map $f$ between metric spaces $\left(X, d_{1}\right)$ and $\left(Y, d_{2}\right)$ is called a $\mathrm{GH}_{\epsilon}$ approximation if $B_{\epsilon}(f(X))=Y$ and $\left|d_{2}\left(f\left(x_{1}\right), f\left(x_{2}\right)\right)-d_{1}\left(x_{1}, x_{2}\right)\right|<\epsilon$ for any $x_{1}, x_{2} \in X$.

- $f:\left(X, d_{1}\right) \longrightarrow\left(Y, d_{2}\right)$ is called a $\varkappa(\delta)$-almost distance preserving map if

$$
\left|1-\frac{|f(x) f(y)|}{|x y|}\right|<\varkappa(\delta) \text { for any } x \neq y \in X ;
$$

and if in addition $f$ is a bijection, $f$ is called a $\varkappa(\delta)$-almost isometry.

- We say that $\bar{f}:\left(X, d_{1}\right) \longrightarrow\left(Y, d_{2}\right)$ is $\nu$-close to $f$ if $|f(x) \bar{f}(x)|<\nu$ for any $x \in X$.

Now we recall the almost isometry theorem stated in [1].

TheOREM 0.1 (TheOREM 9.8 IN [1]). Let $M_{1}$ and $M_{2}$ be two compact $n$ dimensional Alexandrov spaces with the same low curvature bound, and let $h: M_{1} \rightarrow$ $M_{2}$ be a $G H_{\nu}$-approximation. Then for sufficiently small $\delta$ and $\frac{\nu}{R \delta^{3}}$, there exists a $\varkappa\left(\delta, \frac{\nu}{R \delta^{3}}\right)$-almost distance preserving map $\bar{h}: M_{1}(n, \delta, R) \rightarrow M_{2}$ which is $C \nu$-close to $h$.

Note that $\bar{h}$ is a $\varkappa\left(\delta, \frac{\nu}{R \delta^{3}}\right)$-almost isometry between $M_{1}(n, \delta, R)$ and $\bar{h}\left(M_{1}(n, \delta, R)\right)$. And it is not difficult to see that Theorem 0.1 implies the following corollary.

Corollary $0.2([1])$. In Theorem 0.1 if, in addition, each point of $M_{2}$ is $(n, \delta)$ strained, then there exists a $\varkappa(\delta, \nu)$-almost isometry $\bar{h}: M_{1} \longrightarrow M_{2}$ which is $C \nu$-close to $h$.

Theorem 0.1 (and Corollary 0.2) is of importance in connection with the GromovHausdorff convergence of a sequence of $n$-dimensional Alexandrov spaces with the same low curvature bound.

In this paper we give a proof of the following sharpened version of Theorem 0.1.

Theorem A. Let $M_{1}$ and $M_{2}$ be two compact n-dimensional Alexandrov spaces with the same low curvature bound, and let $h: M_{1} \longrightarrow M_{2}$ be a $G H_{\nu}$-approximation. 
Then for sufficiently small $\delta$ and $\nu<\delta^{2} R$, there exists a $\varkappa(\delta)$-almost distance preserving map $\bar{h}: M_{1}(n, \delta, R) \longrightarrow M_{2}$ which is C $\nu$-close to $h$.

Note that in Theorem $0.1 \nu \ll R \delta^{3}$, while we improve that to $\nu<R \delta^{2}$ in Theorem A. This is obtained by sharpening the corresponding estimates in [1].

The proof of Theorem A follows the line of the original proof of Theorem 0.1 in [1]. We first construct $\bar{h}$ (see Section 3) which is the same as in the original proof, and then verify that $\bar{h}$ is an almost distance preserving map (see Section 4). The main difference between our proof and the original proof is in the latter part.

REMARK 0.3. Yamaguchi has proved an almost Lipschitz submersion theorem ([2]), which also implies Corollary 0.2. In [2], the basic idea to construct the almost Lipschitz submersion is to embed an Alexandrov space $M$ with curvature bounded below into $L^{2}(M)$. Compared with it, the basic idea to construct $\bar{h}$ in Theorem 0.1 (and $\mathrm{A}$ ) is to embed $M_{1}(n, \delta, R)$ locally and almost isometrically into the $n$ dimensional Euclidean space (see Theorem 1.1 below).

1. Center of mass and a lemma in [1]. In Theorem 0.1 (and A), the main tool in the construction of $\bar{h}$ is center of mass ([1]). Recall that the center of mass of a point set $Q=\left\{q_{1}, q_{2}, \cdots, q_{l}\right\} \subset \mathbb{R}^{n}$ with weights $W=\left(w_{1}, w_{2}, \cdots, w_{l}\right)$ (where $\sum_{j=1}^{l} w_{j}=1$ and $\left.w_{j} \geqslant 0\right)$ is defined to be

$$
Q_{W}=\sum_{j=1}^{l} w_{j} q_{j}
$$

The construction of the center of mass for a point set in $M$ is based on the following important result.

Theorem 1.1 (Theorem 9.4 in [1]). Let $M$ be an n-dimensional Alexandrov space with curvature bounded below, and let $\left\{\left(a_{i}, b_{i}\right)\right\}_{i=1}^{n}$ be an $(n, \delta)$-strainer at $p \in$ $M$. Then there exist neighborhoods $U$ and $V$ of $p$ and $\left(\left|a_{1} p\right|,\left|a_{2} p\right|, \cdots,\left|a_{n} p\right|\right) \in \mathbb{R}^{n}$ respectively such that

$$
f: U \longrightarrow V \subset \mathbb{R}^{n} \text { defined by } f(q)=\left(\left|a_{1} q\right|,\left|a_{2} q\right|, \cdots,\left|a_{n} q\right|\right)
$$

is a $\varkappa\left(\delta, \delta_{1}\right)$-almost isometry, where $\delta_{1}=\max _{1 \leqslant i \leqslant n}\left\{\left|p a_{i}\right|^{-1},\left|p b_{i}\right|^{-1}\right\} \cdot \operatorname{diam} U$.

In fact, from the proof of Theorem 1.1 (p.35 and 19 in [1]) we can conclude the following refined version of Theorem 1.1.

TheOREm 1.1'. Let $M$ be an $n$-dimensional Alexandrov space with curvature bounded below, and let $\left\{\left(a_{i}, b_{i}\right)\right\}_{i=1}^{n}$ be an $(n, \delta)$-strainer at $p \in M$. Then there exists a constant c such that if a neighborhood $U$ of $p$ satisfies $\max _{1 \leqslant i \leqslant n}\left\{\left|p a_{i}\right|^{-1},\left|p b_{i}\right|^{-1}\right\} \cdot \operatorname{diam} U<$ $c \delta$, then $f: M \longrightarrow \mathbb{R}^{n}$ defined by $f(q)=\left(\left|a_{1} q\right|,\left|a_{2} q\right|, \cdots,\left|a_{n} q\right|\right)$ maps $U \varkappa(\delta)$ isometrically to a domain in $\mathbb{R}^{n}$.

In Theorem $1.1^{\prime}$, the constant $c$ depends only on the dimension $n$, the low bound $k$ of the curvature, and the length $\ell \triangleq \min _{1 \leqslant i \leqslant n}\left\{\left|p a_{i}\right|,\left|p b_{i}\right|\right\}$ if $k<0$ (the larger $\ell$ is, the smaller $c$ is).

In Theorem 1.1 (and 1.1'), if $f(U)$ is convex in $\mathbb{R}^{n}$, then the center of mass of $Q=\left\{q_{1}, q_{2}, \cdots, q_{l}\right\} \subset U$ with weights $W$ is defined to be ([1])

$$
Q_{W}=f^{-1}\left(\sum_{j=1}^{l} w_{j} f\left(q_{j}\right)\right) .
$$


Obviously, $Q_{W}$ depends on the choice of the $(n, \delta)$-strainer at $p$.

Now we formulate the lemma in [1] mentioned at the beginning of the paper.

LEMMA 1.2 (Lemma 9.11 in [1]). Let $p, U,\left\{\left(a_{i}, b_{i}\right)\right\}_{i=1}^{n}$ and $f$ be the same as in Theorem 1.1, and let $\left\{\left(s_{i}, t_{i}\right)\right\}_{i=1}^{n}$ be another $(n, \delta)$-strainer at $p$ with

$$
\delta_{1}=\max \left\{\frac{\operatorname{diam} U}{\min _{i}\left\{\left|p a_{i}\right|,\left|p b_{i}\right|\right\}}, \frac{\max _{i}\left\{\left|p a_{i}\right|,\left|p b_{i}\right|\right\}}{\min _{i}\left\{\left|p s_{i}\right|,\left|p t_{i}\right|\right\}}\right\} .
$$

And let $Q=\left\{q_{1}, \cdots, q_{l}\right\}$ and $R=\left\{r_{1}, \cdots, r_{l}\right\}$ be two point sets in $U$ with

$\max _{j}\left\{\left|q_{j} r_{j}\right|\right\}<(1+\delta) \min _{j}\left\{\left|q_{j} r_{j}\right|\right\}$ and $\left|\max _{j} \tilde{\angle} s_{i} q_{j} r_{j}-\min _{j} \tilde{\angle} s_{i} q_{j} r_{j}\right|<\delta$ for $i=1, \cdots n$.

Assume that $f(U)$ is convex in $\mathbb{R}^{n}$. Then for any weights $W^{1}$ and $W^{2}$ satisfying

$$
\left\|W^{1}-W^{2}\right\|<\delta_{1}
$$

the centers of mass $Q_{W^{1}}$ and $R_{W^{2}}$ (with respect to the strainer $\left\{\left(a_{i}, b_{i}\right)\right\}$ ) satisfy

$$
\left|1-\frac{\left|q_{j} r_{j}\right|}{\left|Q_{W^{1}} R_{W^{2}}\right|}\right|<\varkappa\left(\delta, \delta_{1}\right)
$$

and $\left|\tilde{\angle} s_{i} q_{j} r_{j}-\tilde{\angle} s_{i} Q_{W^{1}} R_{W^{2}}\right|<\varkappa\left(\delta, \delta_{1}\right)$ for $j=1, \cdots, l$ and $i=1, \cdots, n$.

The following counter example shows that this lemma is incorrect.

ExAMPLE 1.3. 1. In fact, if $q_{j}=r_{j}$ for $j=1, \cdots, l$ and $W^{1} \neq W^{2}$, then $Q_{W^{1}} \neq R_{W^{2}}$ and thus

$$
\left|1-\frac{\left|q_{j} r_{j}\right|}{\left|Q_{W^{1}} R_{W^{2}}\right|}\right|=|1-0|>\varkappa\left(\delta, \delta_{1}\right) .
$$

2. If $\left|q_{j} r_{j}\right| \ll|| W^{1}-W^{2} \|$ for all $j$, then " $\left|1-\frac{\left|q_{j} r_{j}\right|}{\left|Q_{W^{1}} R_{W^{2}}\right|}\right|<\varkappa\left(\delta, \delta_{1}\right)$ " does not hold.

As indicated in the example, the condition (1.1) is inadequate for Lemma 1.2 to hold.

2. A modified lemma. In this section we will prove a modified version of Lemma 1.2.

Lemma 2.1. Let $p, U,\left\{\left(a_{i}, b_{i}\right)\right\}_{i=1}^{n}$ and $f$ be the same as in Theorem 1.1', and let $\left\{\left(s_{i}, t_{i}\right)\right\}_{i=1}^{n}$ be another $(n, \delta)$-strainer at $p$ with

$$
\frac{\max _{i}\left\{\left|p a_{i}\right|,\left|p b_{i}\right|\right\}}{\min _{i}\left\{\left|p s_{i}\right|,\left|p t_{i}\right|\right\}}<\varkappa(\delta)
$$

And let $Q=\left\{q_{1}, \cdots, q_{l}\right\}$ and $R=\left\{r_{1}, \cdots, r_{l}\right\}$ be two point sets in $U$. Assume that $f(U)$ is convex in $\mathbb{R}^{n}$, and assume that

$$
\begin{gathered}
\max _{j}\left\{\left|q_{j} r_{j}\right|\right\}<(1+\varkappa(\delta)) \min _{j}\left\{\left|q_{j} r_{j}\right|\right\} \text { and } \\
\left|\max _{j} \tilde{\angle} s_{i} q_{j} r_{j}-\min _{j} \tilde{\angle} s_{i} q_{j} r_{j}\right|<\varkappa(\delta) \text { for } i=1, \cdots, n .
\end{gathered}
$$


Then for any weights $W^{1}$ and $W^{2}$ satisfying

$$
\left\|W^{1}-W^{2}\right\|<\frac{\min _{j}\left\{\left|q_{j} r_{j}\right|\right\}}{\max _{j, j^{\prime}}\left\{\left|r_{j} r_{j^{\prime}}\right|\right\}} \varkappa(\delta),
$$

the centers of mass $Q_{W^{1}}$ and $R_{W^{2}}$ (with respect to the strainer $\left\{\left(a_{i}, b_{i}\right)\right\}$ ) satisfy

$$
\begin{gathered}
\left|1-\frac{\left|q_{j} r_{j}\right|}{\left|Q_{W^{1}} R_{W^{2}}\right|}\right|<\varkappa(\delta) \text { and } \\
\left|\tilde{\angle} s_{i} q_{j} r_{j}-\tilde{\angle} s_{i} Q_{W^{1}} R_{W^{2}}\right|<\varkappa(\delta) \text { for } j=1, \cdots, l \text { and } i=1, \cdots, n .
\end{gathered}
$$

REMARK 2.2. The main change from Lemma 1.2 to Lemma 2.1 is that the condition (1.1) turns into (2.3). Under the condition (2.3), the smaller $\left|q_{j} r_{j}\right|$ is, the smaller ||$W^{1}-W^{2} \|$ is (in particular, if $\left|q_{j} r_{j}\right|=0$, then $W^{1}=W^{2}$ and thus $Q_{W^{1}}=R_{W^{2}}$ ). In the proof of Theorem $\mathrm{A}$, the essential difficulty in verifying that $\bar{h}$ is an almost distance preserving map is to solve the case that $\left|q_{j} r_{j}\right|$ is sufficiently small (see the second paragraph and the proof of Claim 1 in Section 4).

REMARK 2.3. From the original proof of Lemma 1.2 in [1], we can conclude that the following two statements are equivalent:

(1) $\left|\tilde{\angle} a_{i} q_{j} r_{j}-\tilde{\angle} a_{i} q_{j^{\prime}} r_{j^{\prime}}\right|<\varkappa(\delta)$ for $i=1, \cdots, n$;

(2) $\left|\tilde{\angle} s_{i} q_{j} r_{j}-\tilde{L} s_{i} q_{j^{\prime}} r_{j^{\prime}}\right|<\varkappa(\delta)$ for $i=1, \cdots, n$.

For the convenience of readers we give its proof in Appendix.

In the proof of Lemma 2.1, we will use Lemmas 2.4 and 2.6.

LEMMA 2.4. Let $Q=\left\{q_{1}, \cdots, q_{l}\right\}$ and $R=\left\{r_{1}, \cdots, r_{l}\right\}$ be two point sets in $\mathbb{R}^{n}$, and let $W^{i}=\left(w_{1}^{i}, \cdots, w_{l}^{i}\right)$ be two weights with $i=1,2$. Then

$$
\overrightarrow{Q_{W^{1}} R_{W^{2}}}=\sum_{j=1}^{l} w_{j}^{1} \overrightarrow{q_{j} r_{j}}+\sum_{j=1}^{l}\left(w_{j}^{2}-w_{j}^{1}\right) \overrightarrow{r_{j} r_{j}}
$$

for any $j_{0} \in\{1,2, \cdots, l\}$.

Proof. Straightforward computation gives

$$
\begin{aligned}
\overrightarrow{Q_{W^{1}} R_{W_{2}}} & =\sum_{j=1}^{l} w_{j}^{2} r_{j}-\sum_{j=1}^{l} w_{j}^{1} q_{j} \\
& =\sum_{j=1}^{l} w_{j}^{1}\left(r_{j}-q_{j}\right)+\sum_{j=1}^{l}\left(w_{j}^{2}-w_{j}^{1}\right) r_{j} \\
& =\sum_{j=1}^{l} w_{j}^{1} \overrightarrow{q_{j} r_{j}}+\sum_{j=1}^{l}\left(w_{j}^{2}-w_{j}^{1}\right) r_{j}-\sum_{j=1}^{l}\left(w_{j}^{2}-w_{j}^{1}\right) r_{j_{0}} \\
& =\sum_{j=1}^{l} w_{j}^{1} \overrightarrow{q_{j} r_{j}}+\sum_{j=1}^{l}\left(w_{j}^{2}-w_{j}^{1}\right) \overrightarrow{r_{j 0} r_{j}}
\end{aligned}
$$


In order to simplify further discussions, we introduce the following definition.

Definition 2.5. Given point sets $Q=\left\{q_{1}, q_{2}\right\}$ and $R=\left\{r_{1}, r_{2}\right\}$ in $\mathbb{R}^{n}$, we say that $\overrightarrow{q_{1} r_{1}}$ is $\varkappa(\delta)$-almost parallel to $\overrightarrow{q_{2} r_{2}}$ if

$$
\angle\left(\overrightarrow{q_{1} r_{1}}, \overrightarrow{q_{2} r_{2}}\right)<\varkappa(\delta)
$$

and if, in addition,

$$
\left|1-\frac{\left|q_{1} r_{1}\right|}{\left|q_{2} r_{2}\right|}\right|<\varkappa(\delta)
$$

we say that $\overrightarrow{q_{1} r_{1}}$ is $\varkappa(\delta)$-almost equal to $\overrightarrow{q_{2} r_{2}}$.

Lemma 2.6. Let $p, U,\left\{\left(a_{i}, b_{i}\right)\right\}_{i=1}^{n}$ and $f$ be the same as in Lemma 2.1. Then for any points $x_{1}, x_{2}, y_{1}, y_{2} \in U$, the following two statements are equivalent:

(1) $\left|\tilde{\angle} a_{i} x_{1} y_{1}-\tilde{\angle} a_{i} x_{2} y_{2}\right|<\varkappa(\delta)$ for $i=1,2, \cdots, n$;

(2) $\overrightarrow{f\left(x_{1}\right) f\left(y_{1}\right)}$ is $\varkappa(\delta)$-almost parallel to $\overrightarrow{f\left(x_{2}\right) f\left(y_{2}\right)}$.

Lemma 2.6 can be obtained from the original proof of Theorem 0.1 in [1] (we will give its proof in Appendix).

Proof of Lemma 2.1. Note that inequalities (2.1) and (2.2) imply that $\overrightarrow{f\left(q_{j}\right) f\left(r_{j}\right)}$ are $\varkappa(\delta)$-almost equal each other for $j=1, \cdots, l$ (see Remark 2.3, Lemma 2.6 and Theorem 1.1). It then follows from Lemma 2.4 that $\overrightarrow{f\left(Q_{W^{1}}\right) f\left(R_{W^{2}}\right)}$ is $\varkappa(\delta)$-almost equal to $\overrightarrow{f\left(q_{j}\right) f\left(r_{j}\right)}$ for every $j$ (note that $f\left(Q_{W^{1}}\right)=\sum_{j=1}^{l} w_{j}^{1} f\left(q_{j}\right)$ and $f\left(R_{W^{2}}\right)=$ $\sum_{j=1}^{l} w_{j}^{2} f\left(r_{j}\right)$, and $\left.\left\|W^{1}-W^{2}\right\| \cdot \max _{j, j^{\prime}}\left\{\left|r_{j} r_{j^{\prime}}\right|\right\}<\varkappa(\delta) \min _{j}\left\{\left|q_{j} r_{j}\right|\right\}\right)$. Hence, the conclusion of Lemma 2.1 follows from Lemma 2.6, Remark 2.3 and the fact that $f$ is a $\varkappa(\delta)$-almost isometry.

We end this section with a corollary of Remark 2.3 and Lemma 2.6.

Corollary 2.7. Let $p, U,\left\{\left(a_{i}, b_{i}\right)\right\}_{i=1}^{n}, f$ and $\left\{\left(s_{i}, t_{i}\right)\right\}_{i=1}^{n}$ be the same as in Lemma 2.1. Let $\left\{\left(a_{i}^{\prime}, b_{i}^{\prime}\right)\right\}_{i=1}^{n}$ be an $(n, \delta)$-strainer at another point $p^{\prime}$, and let $f^{\prime}$ and $U^{\prime}$ be the map and the neighborhood of $p^{\prime}$ determined by Theorem 1.1' (with respect to $\left.\left\{\left(a_{i}^{\prime}, b_{i}^{\prime}\right)\right\}\right)$. Moreover we assume that $\left\{\left(s_{i}, t_{i}\right)\right\}_{i=1}^{n}$ is also an $(n, \delta)$-strainer at $p^{\prime}$ with

$$
\frac{\max _{i}\left\{\left|p^{\prime} a_{i}^{\prime}\right|,\left|p^{\prime} b_{i}^{\prime}\right|\right\}}{\min _{i}\left\{\left|p^{\prime} s_{i}\right|,\left|p^{\prime} t_{i}\right|\right\}}<\varkappa(\delta) .
$$

Then for any points $x_{1}, x_{2}, y_{1}, y_{2} \in U_{1} \cap U_{2}$, the following two statements are equivalent:

(1) $\overrightarrow{f\left(x_{1}\right) f\left(y_{1}\right)}$ is $\varkappa(\delta)$-almost equal to $\overrightarrow{f\left(x_{2}\right) f\left(y_{2}\right)}$;

(2) $\overrightarrow{f^{\prime}\left(x_{1}\right) f^{\prime}\left(y_{1}\right)}$ is $\varkappa(\delta)$-almost equal to $\overrightarrow{f^{\prime}\left(x_{2}\right) f^{\prime}\left(y_{2}\right)}$.

3. Construction of $\bar{h}$. In this section we construct the $\bar{h}$ in Theorems 0.1 and A (ref. [1]).

Since the closure of $M_{1}(n, \delta, R)$ is compact, we can select $x_{j} \in M_{1}(n, \delta, R)$ with $j=1, \cdots, N_{1}$ such that

$$
\bigcup_{j=1}^{N_{1}} B_{x_{j}}(\delta R) \supset \bigcup_{j=1}^{N_{1}} B_{x_{j}}\left(\frac{1}{3} \delta R\right) \supset M_{1}(n, \delta, R) .
$$


Since $x_{j} \in M_{1}(n, \delta, R)$, there exists an $R$-long $(n, \delta)$-strainer $\left\{\left(s_{i}^{j}, t_{i}^{j}\right)\right\}_{i=1}^{n}$ at $x_{j}$ (with $\left.\min _{i}\left\{\left|x_{j} s_{i}^{j}\right|,\left|x_{j} t_{i}^{j}\right|\right\}>\frac{R}{\delta}\right)$, and thus there exists ([1]) an $(n, \delta)$-strainer $\left\{\left(a_{i}^{j}, b_{i}^{j}\right)\right\}_{i=1}^{n}$ at $x_{j}$ with $\min _{i}\left\{\left|x_{j} a_{i}^{j}\right|,\left|x_{j} b_{i}^{j}\right|\right\}=\min \left\{\frac{2 R}{c}, 1\right\}$ such that

$$
\frac{\max _{i}\left\{\left|x_{j} a_{i}^{j}\right|,\left|x_{j} b_{i}^{j}\right|\right\}}{\min _{i}\left\{\left|x_{j} s_{i}^{j}\right|,\left|x_{j} t_{i}^{j}\right|\right\}}<\frac{2 \delta}{c},
$$

where $c$ is the constant in Theorem 1.1' (if the low bound of the curvature $k<0$, then $c=c(n, k, 1)$ (for $c(n, k, 1)$ please refer to the comments after Theorem 1.1')). Let $f_{j}: M_{1} \longrightarrow \mathbb{R}^{n}$ be the map defined by $f_{j}(q)=\left(\left|q a_{1}^{j}\right|, \cdots,\left|q a_{n}^{j}\right|\right)$. According to Theorem $1.1^{\prime},\left.f_{j}\right|_{B_{x_{j}}(\delta R)}$ is a $\varkappa(\delta)$-almost isometry (note that $\max _{i}\left\{\left|x_{j} a_{i}^{j}\right|^{-1},\left|x_{j} b_{i}^{j}\right|^{-1}\right\}$. $\left.\operatorname{diam}\left(B_{x_{j}}(\delta R)\right)<c \delta\right)$. Then we can select $U_{j}$ with

$$
B_{x_{j}}(3 \delta R / 4) \subset U_{j} \subset B_{x_{j}}(\delta R)
$$

such that $f_{j}\left(U_{j}\right)$ is convex in $\mathbb{R}^{n}$. Obviously,

$$
\left.f_{j}\right|_{U_{j}} \text { is a } \varkappa(\delta) \text {-almost isometry. }
$$

On the other hand, since $h$ is a $\mathrm{GH}_{\nu}$-approximation with $\nu<R \delta^{2}$,

$$
\left\{\left(h\left(a_{i}^{j}\right), h\left(b_{i}^{j}\right)\right)\right\}_{i=1}^{n} \text { and }\left\{\left(h\left(s_{i}^{j}\right), h\left(t_{i}^{j}\right)\right)\right\}_{i=1}^{n} \text { are }(n, 2 \delta) \text {-strainers at } h\left(x_{j}\right) ;
$$

and according to Theorem $1.1^{\prime}$, the map $g_{j}$ defined by $g_{j}(q)=\left(\left|q h\left(a_{1}^{j}\right)\right|, \cdots,\left|q h\left(a_{n}^{j}\right)\right|\right)$ maps $h\left(B_{x_{j}}(\delta R)\right) \varkappa(\delta)$-almost isometrically to a domain in $\mathbb{R}^{n}$. Moreover, without loss of generality, we can assume that $f_{j}\left(U_{j}\right) \subset g_{j}\left(h\left(B_{x_{j}}(\delta R)\right)\right)$. It is clear that

$$
h_{j}=g_{j}^{-1} \circ f_{j} \text { is a } \varkappa(\delta) \text {-almost isometry on each } U_{j} .
$$

Note that for any $x \in U_{j}$,

$$
\begin{aligned}
& \left|h_{j}(x) h(x)\right|=(1+\varkappa(\delta))\left|g_{j}\left(h_{j}(x)\right) g_{j}(h(x))\right| \\
= & (1+\varkappa(\delta))\left|f_{j}(x) g_{j}(h(x))\right| \\
= & (1+\varkappa(\delta)) \sqrt{\left(\left|a_{1}^{j} x\right|-\left|h\left(a_{1}^{j}\right) h(x)\right|\right)^{2}+\cdots+\left(\left|a_{n}^{j} x\right|-\left|h\left(a_{n}^{j}\right) h(x)\right|\right)^{2}} \\
< & (1+\varkappa(\delta)) \sqrt{n} \nu \text { (note that } h \text { is a } \mathrm{GH}_{\nu} \text {-approximation), }
\end{aligned}
$$

i.e. each $h_{j}$ is $C \nu$-close to $h$ on $U_{j}$.

We will use center of mass to glue all these local almost isometries $h_{j}$ to a global one. We first define weight functions ${ }^{1} \phi_{j}: M_{1} \longrightarrow \mathbb{R}$ by

$$
\phi_{j}(x)=\left\{\begin{array}{ll}
1-\frac{2\left|x x_{j}\right|}{\delta R}, & x \in B_{x_{j}}(\delta R / 2) \\
0, & x \in M_{1} \backslash B_{x_{j}}(\delta R / 2)
\end{array} .\right.
$$

Then for an arbitrary point $z \in M_{1}(n, \delta, R)$ we define a sequence $\left\{z_{j}\right\}_{j=1}^{N_{1}} \subset M_{2}$ :

$$
z_{j}=\left\{\begin{array}{ll}
g_{j}^{-1}\left(\frac{\Sigma_{j-1}(z)}{\Sigma_{j}(z)} g_{j}\left(z_{j-1}\right)+\frac{\phi_{j}(z)}{\Sigma_{j}(z)} g_{j}\left(h_{j}(z)\right)\right), & z \in U_{j} \\
z_{j-1}, & z \notin U_{j}
\end{array},\right.
$$

\footnotetext{
${ }^{1}$ The original definition in [1] is $\phi_{j}(x)=\left(1-2\left|x x_{j}\right| /(\delta R)\right)^{N}$ if $x \in B_{x_{j}}(\delta R / 2)$, where $N$ is the multiplicity of the cover $\left\{B_{x_{j}}(\delta R)\right\}$.
} 
where $z_{0}=h(z), \Sigma_{0}(z)=0$ and $\Sigma_{j}(z)=\sum_{l=1}^{j} \phi_{l}(z)$ for $j \geqslant 1$. A basic fact is that

$$
\Sigma_{N_{1}}(z)>\frac{1}{3}(\operatorname{see}(3.0)) \text {. }
$$

Now we define the desired map $\bar{h}: M_{1}(n, \delta, R) \longrightarrow M_{2}$ in Theorem A by

$$
\bar{h}(z)=z_{N_{1}} \text { for any } z \in M_{1}(n, \delta, R) \text {. }
$$

We first show that $\bar{h}$ is $C \nu$-close to $h$. Since each $B_{x_{j}}(\delta R)$ is $\varkappa(\delta)$-almost isometric to a domain in $\mathbb{R}^{n}$, without loss of generality, we can assume that the multiplicity of the cover $\left\{B_{x_{j}}(\delta R)\right\}$ is bounded by a number $N$ depending only on the dimension $n$ (ref. [1]). Since each $h_{j}$ is $C \nu$-close to $h$ and $z$ belongs to at most $N$ pieces of $B_{x_{j}}(\delta R)$, it is easy to see that

$$
\left|h_{j}(z) h_{j^{\prime}}(z)\right|<C \nu \text { and }\left|z_{j} h_{j^{\prime}}(z)\right|<C \nu
$$

and thus

$$
\bar{h} \text { is } C \nu \text {-close to } h \text {. }
$$

In the next section we will verify that $\bar{h}$ is a $\varkappa(\delta)$-almost distance preserving map.

4. Verifying that $\bar{h}$ is an almost distance preserving map. In this section, we will show that $\bar{h}$ (constructed in Section 3 ) is a $\varkappa(\delta)$-almost distance preserving map, i.e. for any $y, z \in M_{1}(n, \delta, R)$,

$$
\left|1-\frac{|\bar{h}(y) \bar{h}(z)|}{|y z|}\right|<\varkappa(\delta) \text { or }|| \bar{h}(y) \bar{h}(z)|-| y z||<\varkappa(\delta)|y z|,
$$

and thus the proof of Theorem A is completed.

We first observe that we only need to consider the case $|y z|<R \delta^{3 / 2}$. In fact, if $|y z| \geqslant R \delta^{3 / 2}$, then || $\bar{h}(y) \bar{h}(z)|-| y z||<C \nu<C R \delta^{2}<|y z| \varkappa(\delta)$ (i.e. (4.1) holds) because $\bar{h}$ is $C \nu$-close to $h$ and $h$ is a $\mathrm{GH}_{\nu}$-approximation.

Without loss of generality, we assume that $\phi_{j}(y)+\phi_{j}(z) \neq 0$ for $1 \leqslant j \leqslant N_{2}$, and $\phi_{j}(y)+\phi_{j}(z)=0$ for $N_{2}<j \leqslant N_{1}$. Note that if $\phi_{j}(y) \neq 0$ (i.e., $y \in B_{x_{j}}(\delta R / 2)$ ), then $z \in B_{x_{j}}(\delta 2 R / 3)$ because $|y z|<R \delta^{3 / 2}$ ( $\delta$ is sufficiently small). Hence

$$
y, z \in B_{x_{j}}(2 \delta R / 3) \text { for } j=1, \cdots, N_{2} \text { and } y, z \notin B_{x_{j}}(\delta R / 2) \text { for } j>N_{2},
$$

which implies that $N_{2} \leqslant N$ (the multiplicity of the cover $\left.\left\{B_{x_{j}}(\delta R)\right\}\right)$. Since $B_{x_{j}}(2 \delta R / 3) \subset B_{x_{j}}(3 \delta R / 4) \subset U_{j}$ (see (3.2)), we have that

$$
\bar{h}(y)=y_{N_{2}} \text { and } \bar{h}(z)=z_{N_{2}} ;
$$

moreover, since each $h_{j}$ is a $\varkappa(\delta)$-almost isometry and $\left|h_{j}(y) h_{j^{\prime}}(y)\right|<C \nu<C R \delta^{2}$ (see $(3.5))$, we have

$$
\left\{h_{1}(y), h_{1}(z), \cdots, h_{N_{2}}(y), h_{N_{2}}(z)\right\} \subset h_{j}\left(U_{j}\right) \text { for any } j=1, \cdots, N_{2} .
$$

Then we can define two sequences $\left\{\bar{y}_{j}\right\}_{j=1}^{N_{2}}$ and $\left\{\bar{z}_{j}\right\}_{j=1}^{N_{2}}$ in $M_{2}$ (which are not introduced in [1]):

$$
\bar{y}_{j}=g_{j}^{-1}\left(\sum_{l=1}^{j} \frac{\phi_{l}(y)}{\Sigma_{j}(y)} g_{j}\left(h_{l}(y)\right)\right) \text { and } \bar{z}_{j}=g_{j}^{-1}\left(\sum_{l=1}^{j} \frac{\phi_{l}(z)}{\Sigma_{j}(z)} g_{j}\left(h_{l}(z)\right)\right) .
$$


Note that $\bar{y}_{j}\left(\right.$ resp. $\left.\bar{z}_{j}\right)$ is the center of mass of $\left\{h_{l}(y)\right\}_{l=1}^{j}$ (resp. $\left.\left\{h_{l}(z)\right\}_{l=1}^{j}\right)$ with weights $W_{y}^{j}=\left(\frac{\phi_{1}(y)}{\Sigma_{j}(y)}, \cdots, \frac{\phi_{j}(y)}{\Sigma_{j}(y)}\right)$ (resp. $W_{z}^{j}=\left(\frac{\phi_{1}(z)}{\Sigma_{j}(z)}, \cdots, \frac{\phi_{j}(z)}{\Sigma_{j}(z)}\right)$ ) with respect to the $(n, 2 \delta)$-strainer $\left\{\left(h\left(a_{i}^{j}\right), h\left(b_{i}^{j}\right)\right)\right\}_{i=1}^{n}$ at $h\left(x_{j}\right)$. And note that

$$
\bar{y}_{j}=y_{j} \text { and } \bar{z}_{j}=z_{j} \text { for } j=1,2 .
$$

Now we give two claims.

Claim $1^{2}$.

$$
|| \bar{y}_{N_{2}} \bar{z}_{N_{2}}|-| y z||<\varkappa(\delta)|y z|
$$

Claim 2.

$$
|| y_{N_{2}} z_{N_{2}}|-| \bar{y}_{N_{2}} \bar{z}_{N_{2}}||<\varkappa(\delta)|y z| .
$$

It is clear that Claims 1 and 2 (together with (4.2)) imply (4.1). Hence, we only need to verify Claims 1 and 2.

The proof of Claim 1. Since $h$ is a $\mathrm{GH}_{\nu}$-approximation with $\nu<R \delta^{2}$, we can obtain that

$$
\begin{array}{r}
\quad\left\{h\left(s_{i}^{j}\right), h\left(t_{i}^{j}\right)\right\}_{i=1}^{n} \text { is an } R \text {-long }(n, 2 \delta) \text {-strainer at } h\left(x_{j^{\prime}}\right) \\
\text { with } \frac{\max _{i}\left\{\left|h\left(x_{j^{\prime}}\right) h\left(a_{i}^{j^{\prime}}\right)\right|,\left|h\left(x_{j^{\prime}}\right) h\left(b_{i}^{j^{\prime}}\right)\right|\right\}}{\min _{i}\left\{\left|h\left(x_{j^{\prime}}\right) h\left(s_{i}^{j}\right)\right|,\left|h\left(x_{j^{\prime}}\right) h\left(t_{i}^{j}\right)\right|\right\}}<\varkappa(\delta)(\text { see }(3.1))
\end{array}
$$

for $j, j^{\prime}=1, \cdots, N_{2}$ (note that $\left|x_{j} x_{j^{\prime}}\right|<2 R \delta$ because $U_{j} \cap U_{j^{\prime}} \neq \emptyset$ ). Then according to Lemma 2.1, Claim 1 follows from the following three properties.

(i) Since each $h_{j}$ is a $\varkappa(\delta)$-almost isometry, we have

$$
\max _{j}\left\{\left|h_{j}(y) h_{j}(z)\right|\right\}<(1+\varkappa(\delta)) \min _{j}\left\{\left|h_{j}(y) h_{j}(z)\right|\right\} .
$$

(ii) For any fixed $j$ (especially for $j=N_{2}$ ),

$$
\left|\max _{l} \tilde{\angle} h\left(s_{i}^{j}\right) h_{l}(y) h_{l}(z)-\min _{l} \tilde{\angle} h\left(s_{i}^{j}\right) h_{l}(y) h_{l}(z)\right|<\varkappa(\delta) \text { for } i=1, \cdots, n .
$$

We will give its proof in Appendix (which is drawn from the original proof of Theorem $0.1([1]))$.

(iii)

$$
\left\|W_{y}^{N_{2}}-W_{z}^{N_{2}}\right\|<\frac{\min _{j}\left\{\left|h_{j}(y) h_{j}(z)\right|\right\}}{\max _{j, j^{\prime}}\left\{\left|h_{j}(z) h_{j^{\prime}}(z)\right|\right\}} \varkappa(\delta) .
$$

In order to prove inequality (4.7), we first give an estimate

$$
\left|\frac{\phi_{l}(y)}{\Sigma_{j}(y)}-\frac{\phi_{l}(z)}{\Sigma_{j}(z)}\right| \leqslant \frac{C|y z|}{\delta R \Sigma_{j}(y)} \text { for } 1 \leqslant l \leqslant j \leqslant N_{2} .
$$

\footnotetext{
${ }^{2}$ The present proof is mainly inspired by this observation.
} 
In fact, we have $\left|\phi_{l}(y)-\phi_{l}(z)\right|=2 \frac{|| z x_{l}|-| y x_{l}||}{\delta R} \leqslant \frac{2|y z|}{\delta R}$ for any $1 \leqslant l \leqslant N_{2}$, and thus

$$
\begin{aligned}
\left|\frac{\phi_{l}(y)}{\Sigma_{j}(y)}-\frac{\phi_{l}(z)}{\Sigma_{j}(z)}\right| & =\frac{1}{\Sigma_{j}(y)}\left|\phi_{l}(y)-\frac{\phi_{l}(z) \Sigma_{j}(y)}{\Sigma_{j}(z)}\right| \\
& =\frac{1}{\Sigma_{j}(y)}\left|\phi_{l}(y)-\phi_{l}(z)-\phi_{l}(z) \frac{\Sigma_{j}(y)-\Sigma_{j}(z)}{\Sigma_{j}(z)}\right| \\
& \leqslant \frac{1}{\Sigma_{j}(y)} \max _{l}\left\{\left|\phi_{l}(y)-\phi_{l}(z)\right|\right\} \cdot N_{2} \\
& \leqslant \frac{C|y z|}{\delta R \Sigma_{j}(y)} .
\end{aligned}
$$

It is clear that inequality (4.7) follows from (4.8) because $\Sigma_{N_{2}}(y)>\frac{1}{3}$ (see (3.4)) and $\left|h_{j}(z) h_{j^{\prime}}(z)\right|<C \nu<C R \delta^{2}$ (see (3.5)).

The proof of Claim 2. Put $\vec{\alpha}_{j}=\overrightarrow{g_{j}\left(y_{j}\right) g_{j}\left(z_{j}\right)}-\overrightarrow{g_{j}\left(\bar{y}_{j}\right) g_{j}\left(\bar{z}_{j}\right)}$ for $j=1, \cdots, N_{2}$. Since each $g_{j}$ is a $\varkappa(\delta)$-almost isometry, Claim 2 is equivalent to

$$
\left|\vec{\alpha}_{N_{2}}\right|<\varkappa(\delta)|y z| \text {. }
$$

SubCLAIM.

(4.10) $\left|\vec{\alpha}_{j}\right| \leqslant \frac{C|y z| \nu}{\delta R \Sigma_{j}(y)}+\frac{\Sigma_{j-1}(y)}{\Sigma_{j}(y)}(1+\varkappa(\delta))\left|\vec{\alpha}_{j-1}\right|+\varkappa(\delta)|y z|$ for $j=2, \cdots, N_{2}$.

It follows from the subclaim that

$$
\begin{aligned}
\left|\vec{\alpha}_{N_{2}}\right| & \leqslant \frac{C|y z| \nu}{\delta R \Sigma_{N_{2}}(y)}+\varkappa(\delta)|y z|+\frac{\Sigma_{N_{2}-1}(y)}{\Sigma_{N_{2}}(y)}(1+\varkappa(\delta))\left|\vec{\alpha}_{N_{2}-1}\right| \\
& \leqslant \frac{C|y z| \nu}{\delta R \Sigma_{N_{2}}(y)}+\varkappa(\delta)|y z|+\frac{\Sigma_{N_{2}-2}(y)}{\Sigma_{N_{2}}(y)}(1+\varkappa(\delta))\left|\vec{\alpha}_{N_{2}-2}\right| \\
& \leqslant \cdots \\
& \leqslant \frac{C|y z| \nu}{\delta R \Sigma_{N_{2}}(y)}+\varkappa(\delta)|y z|+\frac{\Sigma_{2}(y)}{\Sigma_{N_{2}}(y)}(1+\varkappa(\delta))\left|\vec{\alpha}_{2}\right| \\
& <\varkappa(\delta)|y z| \quad\left(\text { note that } \Sigma_{N_{2}}(y)>\frac{1}{3}, \nu<R \delta^{2} \text { and }\left|\vec{\alpha}_{2}\right|=0\right. \text { (see (4.3))). }
\end{aligned}
$$

That is (4.9) holds, so we only need to verify the subclaim.

To simplify notations in the following computations, let us denote $g_{j}(x)$ by $\tilde{x}$ for any $x \in U_{j}$.

Recall that

$$
\tilde{y}_{j}=\frac{\Sigma_{j-1}(y)}{\Sigma_{j}(y)} \tilde{y}_{j-1}+\frac{\phi_{j}(y)}{\Sigma_{j}(y)} \widetilde{h_{j}(y)} \text { and } \widetilde{\bar{y}}_{j}=\sum_{l=1}^{j} \frac{\phi_{l}(y)}{\Sigma_{j}(y)} \widetilde{h_{l}(y)}
$$

$\left(\tilde{z}_{j}\right.$ and $\widetilde{\bar{z}}_{j}$ have the same forms respectively). Through straightforward computation, one can get

$$
\vec{\alpha}_{j}=\frac{\Sigma_{j-1}(y)}{\Sigma_{j}(y)}\left(\widetilde{\widetilde{y}_{j-1} \widetilde{z}_{j-1}}-\sum_{l=1}^{j-1} \frac{\phi_{l}(y)}{\Sigma_{j-1}(y)} \widetilde{\widetilde{h_{l}(y)} \widetilde{h_{l}(z)}}\right)+\sum_{l=1}^{j-1}\left(\frac{\phi_{l}(z)}{\Sigma_{j}(z)}-\frac{\phi_{l}(y)}{\Sigma_{j}(y)}\right) \widetilde{\widetilde{h_{l}(z)} \widetilde{z}_{j-1}} .
$$


Put $\vec{\beta}=\widetilde{\widetilde{y}_{j-1} \widetilde{z}_{j-1}}-\sum_{l=1}^{j-1} \frac{\phi_{l}(y)}{\Sigma_{j-1}(y)} \widetilde{\widetilde{h_{l}(y)} \widetilde{h_{l}(z)}}$ and $\vec{\gamma}=\sum_{l=1}^{j-1}\left(\frac{\phi_{l}(z)}{\Sigma_{j}(z)}-\frac{\phi_{l}(y)}{\Sigma_{j}(y)}\right) \widetilde{\widetilde{h_{l}(z)} \widetilde{z}_{j-1}}$, and thus

$$
\vec{\alpha}_{j}=\frac{\Sigma_{j-1}(y)}{\Sigma_{j}(y)} \vec{\beta}+\vec{\gamma}
$$

It follows from inequalities (4.8) and (3.5) that

$$
|\vec{\gamma}| \leqslant \sum_{l=1}^{j-1} \frac{C|y z|}{\delta R \Sigma_{j}(y)} \cdot C \nu \leqslant \frac{C|y z| \nu}{\delta R \Sigma_{j}(y)} .
$$

In order to estimate $|\vec{\beta}|$, we introduce two points $\bar{z}_{j-1}^{\prime}$ and $z_{j-1}^{\prime}$ such that

$$
\bar{z}_{j-1}^{\prime}=g_{j-1}^{-1}\left(\sum_{l=1}^{j-1} \frac{\phi_{l}(y)}{\Sigma_{j-1}(y)} g_{j-1}\left(h_{l}(z)\right)\right)
$$

and

$$
\overrightarrow{g_{j-1}\left(y_{j-1}\right) g_{j-1}\left(z_{j-1}^{\prime}\right)}=\overrightarrow{g_{j-1}\left(\bar{y}_{j-1}\right) g_{j-1}\left(\bar{z}_{j-1}\right)} .
$$

Now we put

$$
\begin{aligned}
& \vec{\beta}^{1}=\overrightarrow{\widetilde{y}_{j-1} \widetilde{z}_{j-1}}-\overrightarrow{\widetilde{y}_{j-1} \widetilde{z^{\prime}}{ }_{j-1}}, \\
& \vec{\beta}^{2}=\overrightarrow{\widetilde{y}_{j-1}{\widetilde{z^{\prime}}}_{j-1}}-\overrightarrow{\overline{\bar{y}}_{j-1} \widetilde{\bar{z}}_{j-1}}, \\
& \vec{\beta}^{3}=\overrightarrow{\overline{\bar{y}}_{j-1} \widetilde{\bar{z}}_{j-1}}-\overrightarrow{\widetilde{\bar{y}}_{j-1}{\widetilde{\bar{z}^{\prime}}}_{j-1}}, \\
& \vec{\beta}^{4}=\widetilde{\widetilde{\bar{y}}_{j-1}{\widetilde{\bar{z}^{\prime}}}_{j-1}^{\longrightarrow}}-\sum_{l=1}^{j-1} \frac{\phi_{l}(y)}{\Sigma_{j-1}(y)} \widetilde{\widetilde{h_{l}(y)} \widetilde{h_{l}(z)}} .
\end{aligned}
$$

Obviously, $\vec{\beta}=\vec{\beta}^{1}+\vec{\beta}^{2}+\vec{\beta}^{3}+\vec{\beta}^{4}$. In the following, we will analyze $\left|\vec{\beta}^{i}\right|$ one by one. Firstly,

$$
\begin{aligned}
\left|\vec{\beta}^{1}\right| & =\left|\overrightarrow{\widetilde{z}_{j-1}^{\prime} \widetilde{z}_{j-1}}\right|=(1+\varkappa(\delta))\left|z_{j-1}^{\prime} z_{j-1}\right| \\
& =(1+\varkappa(\delta))\left|\overrightarrow{g_{j-1}\left(z_{j-1}^{\prime}\right) g_{j-1}\left(z_{j-1}\right)}\right| \\
& =(1+\varkappa(\delta))\left|\overrightarrow{g_{j-1}\left(y_{j-1}\right) g_{j-1}\left(z_{j-1}\right)}-\overrightarrow{g_{j-1}\left(y_{j-1}\right) g_{j-1}\left(z_{j-1}^{\prime}\right)}\right| \\
(\text { by }(4.13)) & =(1+\varkappa(\delta))\left|\overrightarrow{g_{j-1}\left(y_{j-1}\right) g_{j-1}\left(z_{j-1}\right)}-\overrightarrow{g_{j-1}\left(\bar{y}_{j-1}\right) g_{j-1}\left(\bar{z}_{j-1}\right)}\right| \\
& =(1+\varkappa(\delta))\left|\vec{\alpha}_{j-1}\right| .
\end{aligned}
$$


Secondly,

$$
\begin{aligned}
\left|\vec{\beta}^{3}\right| & =\left|\overrightarrow{\widetilde{\bar{z}}^{\prime}{ }_{j-1} \widetilde{\bar{z}}_{j-1}}\right|=(1+\varkappa(\delta))\left|\bar{z}_{j-1}^{\prime} \bar{z}_{j-1}\right| \\
& =(1+\varkappa(\delta)) \mid \overrightarrow{g_{j-1}\left(\bar{z}_{j-1}^{\prime}\right) g_{j-1}\left(\bar{z}_{j-1}\right) \mid} \\
& =(1+\varkappa(\delta))\left|\sum_{l=1}^{j-1}\left(\frac{\phi_{l}(y)}{\Sigma_{j-1}(y)}-\frac{\phi_{l}(z)}{\Sigma_{j-1}(z)}\right) g_{j-1}\left(h_{l}(z)\right)\right| \\
& =(1+\varkappa(\delta))\left|\sum_{l=1}^{j-1}\left(\frac{\phi_{l}(y)}{\Sigma_{j-1}(y)}-\frac{\phi_{l}(z)}{\Sigma_{j-1}(z)}\right) \overrightarrow{g_{j-1}\left(h_{1}(z)\right) g_{j-1}\left(h_{l}(z)\right)}\right| \\
& \leqslant \frac{C|y z| \nu}{\delta R \Sigma_{j-1}(y)} \quad(\text { similar to getting }(4.12)) .
\end{aligned}
$$

Thirdly, we estimate $\left|\vec{\beta}^{4}\right|$. Note that (4.5) and (4.6) (together with Remark 2.3 and Lemma 2.6) imply that for any $1 \leqslant l, l_{1}, l_{2} \leqslant N_{2}$

$$
\overrightarrow{g_{l}\left(h_{l_{1}}(y)\right) g_{l}\left(h_{l_{1}}(z)\right)} \text { is } \varkappa(\delta) \text {-almost equal to } \overrightarrow{g_{l}\left(h_{l_{2}}(y)\right) g_{l}\left(h_{l_{2}}(z)\right)} \text {, }
$$

and thus

$$
\overrightarrow{g_{j-1}\left(\bar{y}_{j-1}\right) g_{j-1}\left(\bar{z}_{j-1}^{\prime}\right)} \text { is } \varkappa(\delta) \text {-almost equal to } \overrightarrow{g_{j-1}\left(h_{l}(y)\right) g_{j-1}\left(h_{l}(z)\right)} \text {. }
$$

Then due to (4.4), we can apply Corollary 2.7 to get that

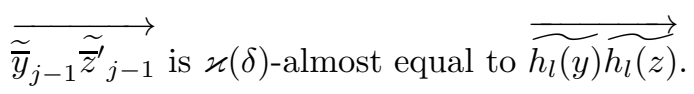

On the other hand, by (4.14)

$$
\sum_{l=1}^{j-1} \frac{\phi_{l}(y)}{\Sigma_{j-1}(y)} \widetilde{\widetilde{h_{l}(y)} \widetilde{h_{l}(z)}} \text { is } \varkappa(\delta) \text {-almost equal to } \widetilde{\widetilde{h_{l}(y)} \widetilde{h_{l}(z)}} .
$$

It therefore follows that

$$
\left|\vec{\beta}^{4}\right|<\varkappa(\delta)\left|\widetilde{h_{l}(y)} \widetilde{h_{l}(z)}\right|=\varkappa(\delta)|y z|
$$

Finally, we estimate $\left|\vec{\beta}^{2}\right|$. Note that it follows from (4.15) that $\mid \overrightarrow{\widetilde{\bar{y}}_{j-1}{\widetilde{\bar{z}^{\prime}}}_{j-1} \mid}<$ $\varkappa(\delta)|y z|$, and thus

$$
\left|\widetilde{\bar{y}}_{j-1} \widetilde{\bar{z}}_{j-1}\right| \leqslant\left|\vec{\beta}^{3}\right|+\left|\widetilde{\bar{y}}_{j-1}{\widetilde{\bar{z}^{\prime}}}_{j-1}\right|<\frac{C|y z| \nu}{\delta R \Sigma_{j-1}(y)}+\varkappa(\delta)|y z| .
$$

On the other hand, according to Corollary 2.7, it follows from (4.13) that

$$
\overrightarrow{\widetilde{y}_{j-1}{\widetilde{z^{\prime}}}_{j-1}} \text { is } \varkappa(\delta) \text {-almost equal to } \overrightarrow{\widetilde{\bar{y}}_{j-1} \widetilde{\bar{z}}_{j-1}} \text {. }
$$

Therefore, we have

$$
\left|\vec{\beta}^{2}\right| \leqslant \varkappa(\delta)\left|\widetilde{\bar{y}}_{j-1} \widetilde{\bar{z}}_{j-1}\right| \leqslant \varkappa(\delta)\left(\frac{C|y z| \nu}{\delta R \Sigma_{j-1}(y)}+\varkappa(\delta)|y z|\right) .
$$

Now we conclude that

$$
|\vec{\beta}| \leqslant\left|\vec{\beta}^{1}\right|+\left|\vec{\beta}^{2}\right|+\left|\vec{\beta}^{3}\right|+\left|\vec{\beta}^{4}\right|<(1+\varkappa(\delta))\left|\vec{\alpha}_{j-1}\right|+\frac{C|y z| \nu}{\delta R \Sigma_{j-1}(y)}+\varkappa(\delta)|y z| .
$$

Since $\vec{\alpha}_{j}=\frac{\Sigma_{j-1}(y)}{\Sigma_{j}(y)} \vec{\beta}+\vec{\gamma}$ (see (4.11)), it is clear that the estimates of $|\vec{\beta}|$ and $|\vec{\gamma}|$ (see (4.12)) imply (4.10) (i.e. the Subclaim holds, so the whole proof is finished). 
5. Appendix. In Appendix, we give the proofs of Remark 2.3, Lemma 2.6 and (4.6). In the proof of Remark 2.3, we will use a result contained in Lemma 5.6 in [1].

Lemma 5.1. Let $p, q, r, s \in M$. For sufficiently small $\delta$, if $|q s|<\delta \cdot \min \{|p q|,|r q|\}$ and $\tilde{\angle} p q r>\pi-\delta$, then ${ }^{3}|\tilde{\angle} p q s-\angle p q s|<\varkappa(\delta)$ and $|\tilde{L} r q s-\angle r q s|<\varkappa(\delta)$.

Proof of Remark 2.3. According to Lemma 5.1, the conclusion in Remark 2.3 is equivalent to

$$
\left|\angle a_{i} q_{j} r_{j}-\angle a_{i} q_{j^{\prime}} r_{j^{\prime}}\right|<\varkappa(\delta) \Longleftrightarrow\left|\angle s_{i} q_{j} r_{j}-\angle s_{i} q_{j^{\prime}} r_{j^{\prime}}\right|<\varkappa(\delta) \text { for } i=1, \cdots, n \text {. }
$$

Using the law of cosine, it is not difficult to conclude that

$$
\left|\tilde{L} u q_{j} v-\tilde{L} u q_{j^{\prime}} v\right|<\varkappa(\delta) \text { for } u \in\left\{s_{i}, t_{i}\right\}_{i=1}^{n} \text { and } v \in\left\{a_{i}, b_{i}\right\}_{i=1}^{n} \text {. }
$$

By Lemma 5.1 again,

$$
\left|\angle u q_{j} v-\angle u q_{j^{\prime}} v\right|<\varkappa(\delta)
$$

Now we consider the direction space $\Sigma_{q_{j}}$ at $q_{j}$. In the situation here, $\Sigma_{q_{j}}$ is $\varkappa(\delta)$ almost isometric to a unit sphere $\mathbb{S}^{n-1}$ (see Theorem 9.5 in [1]). Denote by $\bar{a}_{i} \in \Sigma_{q_{j}}$ (resp. $\bar{s}_{i}$ and $\bar{r}_{j}$ ) the directions of geodesics $q_{j} a_{i}$ (resp. $q_{j} s_{i}$ and $q_{j} r_{j}$ ) for $i=1, \cdots, n$. Note that $\left|\bar{a}_{i} \bar{a}_{i^{\prime}}\right|=\frac{\pi}{2} \pm \varkappa(\delta)$ and $\left|\bar{s}_{i} \bar{s}_{i^{\prime}}\right|=\frac{\pi}{2} \pm \varkappa(\delta)$ for $i \neq i^{\prime}$ (i.e. $\left\{\bar{a}_{i}\right\}_{i=1}^{n}$ and $\left\{\bar{s}_{i}\right\}_{i=1}^{n}$ roughly correspond to two orthogonal $n$-frames of $\left.\mathbb{S}^{n-1}\right)$. Similarly, we also consider $\Sigma_{q_{j}^{\prime}}$. Then it is not difficult to see that inequality (5.2) implies (5.1).

Proof of Lemma 2.6. We first note that

$$
\begin{aligned}
& \left|\tilde{\angle} a_{i} x_{1} y_{1}-\tilde{\angle} a_{i} x_{2} y_{2}\right|<\varkappa(\delta) \\
\Longleftrightarrow & \left|\cos \tilde{\angle} a_{i} x_{1} y_{1}-\cos \tilde{\angle} a_{i} x_{2} y_{2}\right|<\varkappa(\delta) \\
\Longleftrightarrow & \left|\frac{\left|a_{i} x_{1}\right|^{2}+\left|x_{1} y_{1}\right|^{2}-\left|a_{i} y_{1}\right|^{2}}{2\left|a_{i} x_{1}\right| \cdot\left|x_{1} y_{1}\right|}-\frac{\left|a_{i} x_{2}\right|^{2}+\left|x_{2} y_{2}\right|^{2}-\left|a_{i} y_{2}\right|^{2}}{2\left|a_{i} x_{2}\right| \cdot\left|x_{2} y_{2}\right|}\right|<\varkappa(\delta) \\
\Longleftrightarrow & \left|\frac{\left|a_{i} x_{1}\right|-\left|a_{i} y_{1}\right|}{\left|x_{1} y_{1}\right|}-\frac{\left|a_{i} x_{2}\right|-\left|a_{i} y_{2}\right|}{\left|x_{2} y_{2}\right|}\right|<\varkappa(\delta) \\
\Longleftrightarrow & \left|\frac{\left|a_{i} x_{1}\right|-\left|a_{i} y_{1}\right|}{\left|f\left(x_{1}\right) f\left(y_{1}\right)\right|}-\frac{\left|a_{i} x_{2}\right|-\left|a_{i} y_{2}\right|}{\left|f\left(x_{2}\right) f\left(y_{2}\right)\right|}\right|<\varkappa(\delta)(f \text { is a } \varkappa(\delta) \text {-almost isometry)},
\end{aligned}
$$

where the second " $\Longleftrightarrow$ " is due to that the neighborhood $U$ in the lemma is sufficiently small. Recall that $f(x)=\left(\left|a_{1} x\right|,\left|a_{2} x\right|, \cdots,\left|a_{n} x\right|\right)$. Hence, $\mid \tilde{L} a_{i} x_{1} y_{1}-$ $\tilde{Z} a_{i} x_{2} y_{2} \mid<\varkappa(\delta)$ for $i=1,2, \cdots, n \Longleftrightarrow \angle\left(\overrightarrow{f\left(x_{1}\right) f\left(y_{1}\right)}, \overrightarrow{f\left(x_{2}\right) f\left(y_{2}\right)}\right)<\varkappa(\delta)$.

\footnotetext{
${ }^{3} \angle p q s$ is the angle between geodesics $q p$ and $q s$ at $q$, which is well defined by $\lim _{x, y \longrightarrow q} \tilde{\angle} x q y$ with $x \in q p$ and $y \in q s$.
} 
Proof of (4.6). For any $1 \leqslant l_{1}, l_{2} \leqslant N_{2}$ and $u \in\left\{s_{i}^{j}, t_{i}^{j}\right\}_{i=1}^{n}$, we notice that

$$
\begin{aligned}
& \text { the inequality (4.6) } \\
& \Longleftrightarrow\left|\tilde{\angle} h(u) h_{l_{1}}(y) h_{l_{1}}(z)-\tilde{\angle} h(u) h_{l_{2}}(y) h_{l_{2}}(z)\right|<\varkappa(\delta) \\
& \text { (obviously) } \Longleftrightarrow\left|\tilde{\angle} h(u) h_{l}(y) h_{l}(z)-\tilde{\angle} u y z\right|<\varkappa(\delta) \text { for } l=1, \cdots, N_{2} \\
&\left(\text { by Lemma 5.1) } \Longleftrightarrow\left|\angle h(u) h_{l}(y) h_{l}(z)-\angle u y z\right|<\varkappa(\delta)\right. \\
& \text { (by Lemma 5.1) } \Longleftrightarrow\left|\angle h\left(a_{i}^{l}\right) h_{l}(y) h_{l}(z)-\angle a_{i}^{l} y z\right|<\varkappa(\delta) \\
&\text { (similar to }(5.3)) \Longleftrightarrow\left|\tilde{\angle} h\left(a_{i}^{l}\right) h_{l}(y) h_{l}(z)-\tilde{\angle} a_{i}^{l} y z\right|<\varkappa(\delta) \\
&\left|\frac{\left.\left|h\left(a_{i}^{l}\right) h_{l}(y)\right|-\mid h\left(a_{i}^{l}\right) h_{l}(z)\right) \mid}{\left|h_{l}(y) h_{l}(z)\right|}-\frac{\left|a_{i}^{l} y\right|-\left|a_{i}^{l} z\right|}{|y z|}\right|<\varkappa(\delta),
\end{aligned}
$$

where the last inequality holds because $\left|h\left(a_{i}^{l}\right) h_{l}(y)\right|=\left|a_{i}^{l} y\right|,\left|h\left(a_{i}^{l}\right) h_{l}(z)\right|=\left|a_{i}^{l} z\right|$ (recall that $\left.h_{l}=g_{l}^{-1} \circ f_{l}\right)$ and $h_{l}$ is a $\varkappa(\delta)$-almost isometry.

Now we only need to verify the third ' $\Longleftrightarrow$ ' in (5.4). Similar to getting inequality (5.2), we can obtain that for any $v \in\left\{a_{i}^{l}, b_{i}^{l}\right\}_{i=1}^{n}$

$$
\left|\angle h(u) h_{l}(y) h(v)-\angle u y v\right|<\varkappa(\delta) \text {. }
$$

Then we can use the same argument as in the end of the proof of Remark 2.3 to conclude that the third ' $\Sigma_{h_{l}(y)}$ are $\varkappa(\delta)$-almost isometric to $\left.\mathbb{S}^{n-1}\right)$.

Acknowledgement. The authors are grateful to Professor Yuri Burago for his numerous constructive comments/suggestions on this paper.

\section{REFERENCES}

[1] Yu. Burago, M. Gromov, and G. Perel'man, A. D. Alexandrov spaces with curvature bounded below, Russian Math. Surveys, 47:2 (1992), pp. 1-58.

[2] T. Yamaguchi, A convergence theorem in the geometry of Alexandrov spaces, 1996, preprint. 